\title{
Spectroscopic Evidence for a New Type of Bonding between a Thioether Radical Cation and a Phenyl Group
}

\author{
Nicolas P.-A. Monney, ${ }^{\dagger}$ Thomas Bally, ${ }^{,}{ }^{\dagger}$ Ganga S. Bhagavathy, ${ }^{\ddagger}$ and \\ Richard S. Glass ${ }^{\star, \ddagger}$ \\ Department of Chemistry, University of Fribourg, CH-1700, Fribourg, Switzerland, and \\ the Department of Chemistry and Biochemistry, The University of Arizona, Tucson, \\ Arizona 85721, United States
}

Thomas.Bally@unifr.ch;rglass@email.arizona.edu

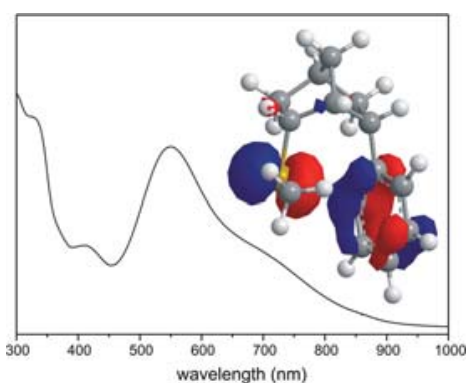

The oxidation potential of thioethers constrained to be near aromatic rings is lowered, due to an antibonding interaction between the p-type sulfur lone pair with the neighboring phenyl $\pi$-system which on removal of an electron becomes a new kind of 3-electron $\mathrm{S}: \therefore \pi$ bonding that reveals itself in the photoelectron spectrum and by an electronic transition involving the orbitals participating in the $S \therefore \pi$ bond.

Methionine is one of the most easily oxidized amino acids, and its thioether functional group has a high susceptibility to be attacked by free radicals. ${ }^{1}$ It has been suggested to play a role in the pathogenesis of neurodegenerative diseases such as Parkinson's ${ }^{2}$ and Alzheimer's ${ }^{3}$ disease. Also, methionine has been postulated to be involved in long-range electron transfer through proteins using a multistep hopping process, ${ }^{4}$ a process that is, however, not thermodynamically feasible unless the incipient thioether radical cations are stabilized by some neighboring moieties.

An unusual property of the radical cations of dialkyl sulfides is their propensity to form two-center, three-

\footnotetext{
$\dagger$ The University of Fribourg.

\$ The University of Arizona.

(1) (a) Vogt, W. Free Radical Biol. Med. 1995, 18, 93-105. (b) Bobrowski, K.; Houée-Levin, C.; Marciniak, B. Chimia 2008, 62, 728734.

(2) Wassef, R.; Haenold, R.; Hansel, A.; Brot, N.; Heinemann, S. H.; Hoshi, T. J. Neurosci. 2007, 27, 12808-12816.

(3) Butterfield, D. A.; Boyd-Kimball, D. Biochim. Biophys. Acta 2005, 1703, 149-156.

(4) Wang, M.; Gao, P.; Müller, P.; Giese, B. Angew. Chem., Int. Ed. 2009, 48, 4232-4234.
}

electron bonds with the nonbonding electrons of the neutral precursor, or with those of another heteroatom ${ }^{5}$ ( $\mathrm{X}$ in Scheme 1, left side). Such complexes distinguish themselves by broad $\sigma \rightarrow \sigma^{*}$ absorption bands which usually lie in the visible range. ${ }^{5 \mathrm{a}}$

Using model compounds where a methylthio and an amide moiety are attached to a norbornane skeleton, it was shown that such $[\mathrm{S} \therefore \mathrm{X}]$ complexation leads indeed to a decrease of the oxidation potential of the thioether, ${ }^{6}$ as shown in Scheme 1. Hence, such interaction may account for the enhanced rate of electron transfer in model peptides where such interactions are possible.

This paper provides experimental evidence that this propensity of sulfur radical cations to stabilize themselves by interacting with neighboring lone pairs can be extended

(5) (a) Asmus, K.-D. In Sulfur-Centered Reactive Intermediates in Chemistry and Biology; Chatgilialoglu, C., Asmus, K.-D., Eds.; NATO ASI Series Vol. 197; Plenum: New York, 1990; pp 155-172. (b) Fourré, I.; Silvi, B. Heteroatom Chem. 2007, 18, 135-160. (c) Lanzalunga, O.; Lapi, A. J. Sulfur Chem. 2012, 33, 101-129.

(6) Glass, R. S.; Hug, G. L.; Schöneich, C.; Wilson, G. S.; Kuznetsova, L.; Lee, T.-K.; Ammam, M.; Lorance, E.; Nauser, T.; Nichol, G. S.; Yamamoto, T. J. Am. Chem. Soc. 2009, 131, 13791-13805. 
to aromatic $\pi$-systems. Note that this "through-space" interaction is distinct from the well-known aromatic $\pi$-delocalization of sulfur-centered radical cations directly attached to an aromatic ring. ${ }^{7}$ It has also been reported that the radical cations of aromatic sulfides can form $2 \mathrm{c}, 3 \mathrm{e}$ $[\mathrm{S} \therefore \mathrm{S}]$ as well as $[\pi \therefore \pi]$ dimers. $^{8}$

Initial indications that aromatic compounds might stabilize sulfur radical cations were obtained by Werst ${ }^{9}$ who showed that dialkyl sulfide radical cations generated in toluene did not aggregate to form $[\mathrm{S} \therefore \mathrm{S}]$ bonds, leading him to conclude that the toluene stabilized in some way the sulfur radical cations. Morgan et al. ${ }^{10}$ were the first to speculate that such interaction may be important in biological electron transfer, based on the observation that a structural motif with alternating sulfur side chains (methionine, cysteine) and aromatic ones (phenylalanine, tyrosine, tryptophan) was found in the crystal structures of eight small globular proteins.

Scheme 1. Formation of $[\mathrm{S} \therefore \mathrm{X}]+$ (left) and $[\mathrm{S} \therefore \pi]+$ (right) Complexes

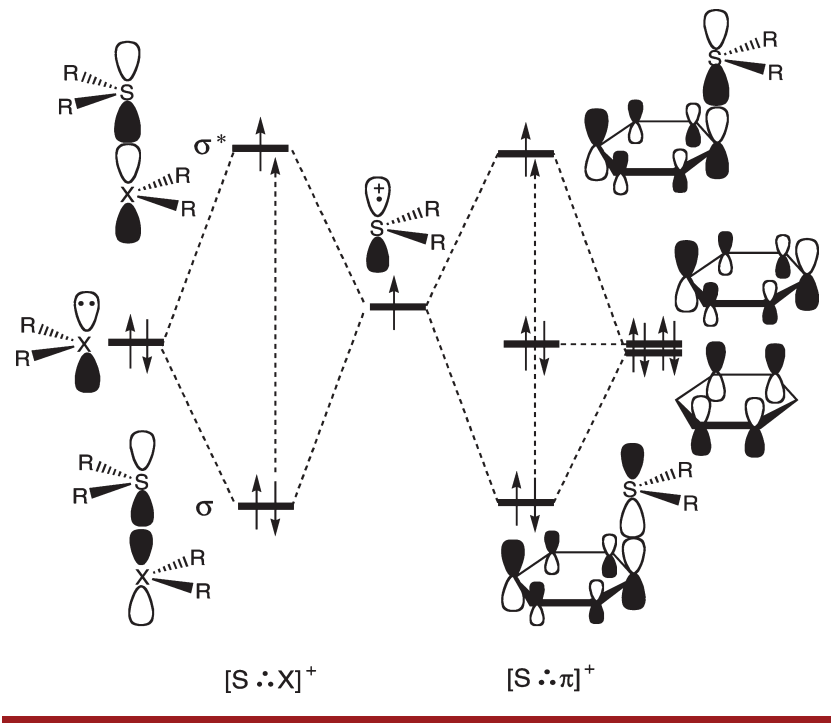

Furthermore, previous studies ${ }^{11}$ on $\mathbf{1}$, in which a thioether and an aromatic ring are both endo, showed that it undergoes electrochemical oxidation at a potential of $300 \mathrm{mV}$ below that in the isomer where the phenyl group is exo, i.e. where through space interaction is geometrically precluded. In a recent theoretical study Chen et al. ${ }^{12}$ suggested that there is indeed an electronic interaction between sulfur-centered radical cations and aromatic

(7) (a) Baciocchi, E.; Gerini, M. F. J. Phys. Chem. A 2004, 108, 23322338. (b) Dondi, V.; Barone, V.; Buttafava, O.; Lanzalunga, O. Tetrahedron Lett. 2011, 52, 4097-4102.

(8) Yokoi, H.; Hatta, A.; Ishiguro, K.; Sawaki, Y. J. Am. Chem. Soc. 1998, 120, 12728-12733.

(9) Werst, D. W. J. Phys. Chem. 1992, 96, 3640-3646.

(10) Morgan, R. S.; Tatsch, D. E.; Gushard, R. H.; McAdon, J. M.; Warme, P. K. Int. J. Pept. Protein Res. 1978, 11, 209-217.

(11) Chung, W.; Ammam, M.; Gruhn, N.; Nichol, G. S.; Singh, W. P.; Wilson, G. S.; Glass, R. S. Org. Lett. 2009, 11, 397-400.

(12) Chen, X.; Tao, Y.; Li, J.; Dai, H.; Sun, W.; Huang, X.; Wei, Z. J. Phys. Chem. C 2012, 116, 19682-19688. $\pi$-systems. They optimized the structures of various $[\mathrm{S} \therefore \pi]^{+}$ complexes and analyzed the MOs that are involved in their formation, and they showed that the complexes involve a multicenter three-electron bond (Scheme 1, right side).<smiles>CSC1CC2CC(c3ccccc3)C1C2</smiles>

1<smiles>c1ccc(C2CC3CCC2C3)cc1</smiles>

2

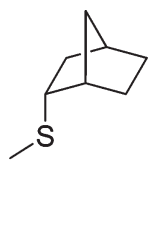

3
The present paper provides the first direct experimental evidence for this new type of $[\mathrm{S} \therefore \pi]$ bonding in the radical cation of $\mathbf{1}$. We report spectroscopic signatures for this interaction, validated by theoretical calculations. The photoelectron (PE) spectra of the constituent components $\mathbf{2}$ and $\mathbf{3}$ and the $\mathrm{UV}$-vis electronic absorption (EA) of the corresponding radical cations were also measured to highlight the spectroscopic features that are characteristic of the $[\mathrm{S}: \pi]$ interaction.

Compound $\mathbf{1}$ was synthesized as described by Chung et al. ${ }^{11}$ Compound $\mathbf{2}$ was made by hydrogenation of commercially available 2-phenyl-2-norbornene. ${ }^{13}$ The synthesis of $\mathbf{3}$ was achieved by a Diels-Alder reaction of cyclopentadiene with methyl vinyl sulfone, followed by catalytic hydrogenation. ${ }^{14}$ The resulting 2-endo-methanesulfonylnorbornane was then reduced to the thioether $\mathbf{3}$ and purified by column chromatography. ${ }^{15}$

Radical cations were formed by $\gamma$-irradiation of glassy matrices made from $5 \mathrm{mM}$ solutions of $\mathbf{1}, \mathbf{2}$, or $\mathbf{3}$ in a $1: 1$ (v/v) mixture of $\mathrm{CFCl}_{3}$ and $\mathrm{CF}_{2} \mathrm{Br}-\mathrm{CF}_{2} \mathrm{Br}$. The solutions were frozen at $77 \mathrm{~K}$ and irradiated in homemade cuvettes with a ca. $1 \mathrm{~mm}$ optical path length. EA spectra were recorded in a cryostat (Oxford Instrument) by a PerkinElmer Lambda 900 spectrometer. The PE spectra were measured on a modified Perkin-Elmer PS18 He I photoelectron spectrometer.

To analyze the spectra (TD)-DFT calculations were performed using the double hybrid functional B2PLYP ${ }^{16}$ with the cc-pVDZ basis set. ${ }^{17}$ All structures were optimized, and the stationary points were identified using the Gaussian 09 suite of programs. ${ }^{18}$ Excited state TD-B2PLYP calculations were carried out using the ORCA program suite. ${ }^{19}$ The first ionization energies in the PE spectra were calculated by the $\triangle \mathrm{SCF}$ method; i.e., the vertical energy difference is taken between the radical cation and the

(13) Kropp, P. J. J. Am. Chem. Soc. 1973, 95, 4611-4619.

(14) Philips, J. C.; Oku, M. J. Org. Chem. 1972, 37, 4479-4480.

(15) Glass, R. S.; Sabahi, M.; Singh, W .P. J. Org. Chem. 1992, 57 2683-2688.

(16) Neese, F.; Schwabe, T.; Grimme, S. J. Chem. Phys. 2007, 126 (124115), 1-14.

(17) The popular B3LYP method is not capable of modeling correctly the mid- and long-range correlation effects that prevail in radical cation complexes, while the MP2 treatment included in the B2PLYP method has been shown to yield a better description of these interactions (see, e.g., Bloch-Mechkour, A.; Bally, T.; Marcinek, A. J. Phys. Chem. A 2011, 115, 7700-7708). Results obtained with various other methods are listed in the Supporting Information.

(18) Frisch, M. J. et al. Gaussian 09, Revision C.1; Gaussian, Inc.; Wallingford, CT, 2009.

(19) Neese, F. ORCA, an ab initio, density functional and semiempirical program package; University Bonn: 2007. 
neutral at the optimized geometry of the latter. Higher ionization potentials were calculated by TD-B2PLYP, adding the energy of the excited states to the $\triangle \mathrm{SCF}$ energy.

We found that neutral $\mathbf{1}$ has three potential energy minima which differ in the conformation of the methylthio group (Scheme 2, top). The $\mathrm{S}-\mathrm{Me}$ bond can either be approximately parallel to the phenyl group (1a and 1c) or perpendicular to it (1) $)$. In conformer 1a the SMe group points away from the norbornyl moiety, while in $\mathbf{1 c}$ it is tucked under it. The rotational barriers between conformers $\mathbf{1 b}$ and $\mathbf{1 a}$, as well as between $\mathbf{1 b}$ and $\mathbf{1 c}$, were both found to be so small that free equilibration of the three conformers takes place in solution, even at low temperatures. According to the relative free energies shown in Scheme 2, about half of the molecules adopt conformation 1b, and half conformation 1a.

Scheme 2. Conformations of $\mathbf{1}$ and $\mathbf{1}^{\mathbf{}+}$ (relative energies in $\mathrm{kcal} / \mathrm{mol}$ )

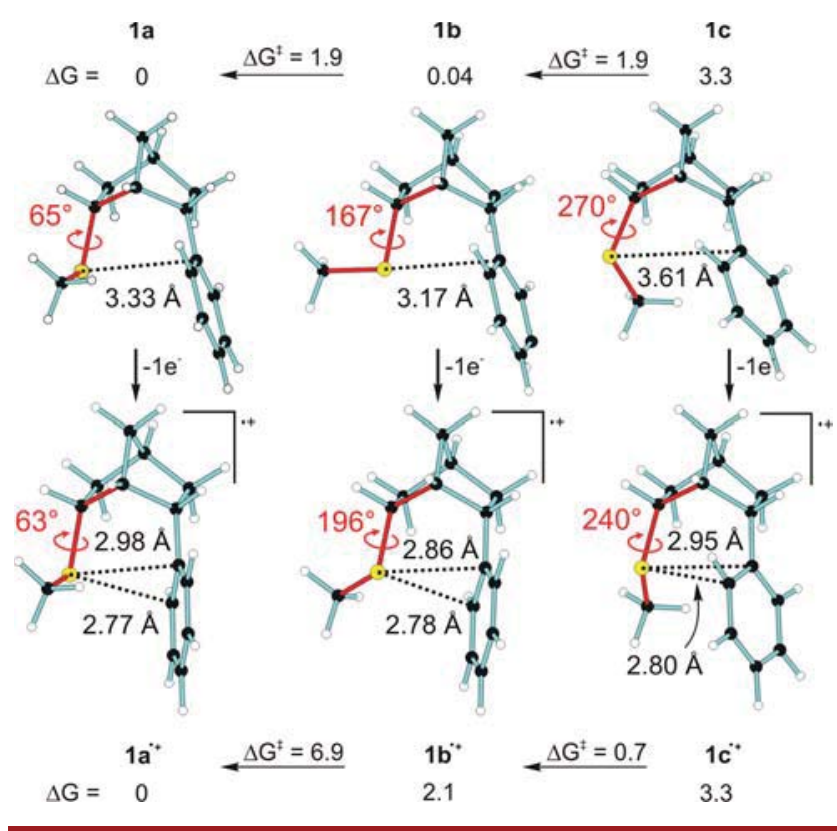

The photoelectron spectra of $\mathbf{1}, \mathbf{2}$, and $\mathbf{3}$ are shown in Figure 1. The PE spectrum of $\mathbf{1}$ was previously published and analyzed, ${ }^{11}$ but without accounting for the conformer distribution of $\mathbf{1}$. All three spectra are composed of two regions: above $9.75 \mathrm{eV}$, ionizations arise from $\sigma$-type orbitals located on the norbornyl moiety.

Below $9.5 \mathrm{eV}$, ionizations from the sulfur p lone pair (3), the two highest phenyl $\pi$-MOs (2), or a mixture of both (1) are observed. The varying band intensities in the spectrum of 1 indicate the presence of at least two conformers in a ca. $1: 2$ ratio, in qualitative agreement with the above prediction of two predominant conformers, $\mathbf{1 a}$ and $\mathbf{1 b}$.

(20) Evidently, modelling of the PE spectrum of $\mathbf{1}$ in terms of binary MO-interacations is insufficient to capture all of its features, although it provides evidence for the interaction between the $\mathrm{S}$ lone pair and the MOs of the phenyl ring. Also, the B2PLYP calculations, which are quite successful at modeling $\mathbf{2}$ and $\mathbf{3}$, are imperfect in modelling the ionic states of $\mathbf{1}$ which involve, next to the occupied fragment MOs, the virtual-MOs and the MOs of the norbornane skeleton.
The orbital interactions that lead to the PE spectrum of the predominant conformer, 1a, are indicated by the dashed lines in Figure 1. Figure S1 (see Supporting Information (SI)) reproduces this interaction diagram, using the calculated orbitals and the experimental state energies (cf. Table 1). The interaction of the p lone pair on the sulfur atom of $\mathbf{3}$ with the $\pi$-HOMO of the phenyl ring of $\mathbf{2}$ leads to a splitting of the two levels in $\mathbf{1}$. The subjacent $\pi$-MO of $\mathbf{2}$ is also destabilized on going from $\mathbf{2}$ to $\mathbf{1}$, presumably through interaction with lower-lying $\sigma$-MOs.

Superimposed on the spectrum of conformer 1a is the weaker one of $\mathbf{1 b}$ where the splitting of the $\pi$-MOs is much smaller (cf. red bars in Figure 1) because there is very little interaction between the $\mathrm{p}$ lone pair on sulfur and the phenyl ring (see Figure S2 in the SI). ${ }^{20}$

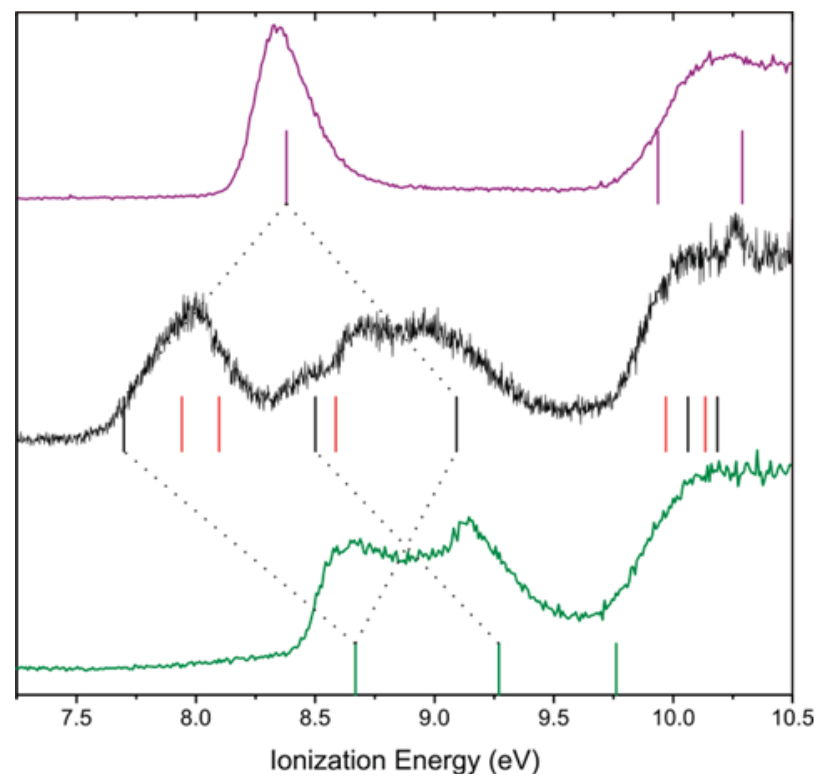

Figure 1. PE spectra of $\mathbf{1}$ (black), $\mathbf{2}$ (green), and $\mathbf{3}$ (purple). The bars represent the energies of the radical cation states calculated at the neutral geometries of $\mathbf{1 a}$ (black bars), $\mathbf{1 b}$ (red bars), $\mathbf{2}$ (green bars), and $\mathbf{3}$ (purple bars) by TD-B2PYLP, relative to the ground states of the radical cations, the energy of which was evaluated by $\triangle \mathrm{SCF}$ calculations. All energies are shifted by $0.25 \mathrm{eV}$ to bring them into better accord with experiment.

After one-electron oxidation of $\mathbf{1 a}, \mathbf{1 b}$, and $\mathbf{1 c}$, the $[\mathrm{S} \therefore \pi]^{+}$complexes $\mathbf{1 a}^{\bullet+}, \mathbf{1}^{\bullet+}$, and $\mathbf{1} \mathbf{c}^{\bullet+}$, respectively, are formed (Scheme 2). As the barrier for the conversion of

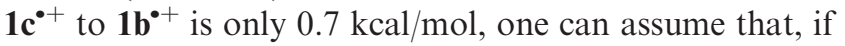
any $\mathbf{1} \mathbf{c}^{\mathbf{}+}$ is formed, it decays instantly to $\mathbf{1 b}^{\mathbf{}}$. Therefore, we only have to consider the two conformers $\mathbf{1 a}^{\mathbf{\bullet}}$ and $\mathbf{1} \mathbf{b}^{\mathbf{}}+$ in the discussion of the EA spectrum of this radical cation, which is shown in Figure 2. As it turns out, the excited state

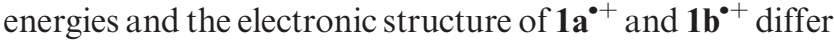
insignificantly, so we will base our discussion on $\mathbf{1 a}^{\mathbf{}^{+}}$, which is formed from the prevailing conformer $\mathbf{1 a}$ and which is furthermore more stable than $\mathbf{1} \mathbf{b}^{\mathbf{}}$.

What becomes very clearly evident from the calculations is that the distance between the S-atom and the plane of the 
Table 1. Lowest Ionization Energies for $\mathbf{1 a}, \mathbf{1 b}, \mathbf{2}$, and $\mathbf{3}$ (in eV)

\begin{tabular}{lll}
\hline compd & \multicolumn{1}{c}{ experimental } & \multicolumn{1}{c}{ calculated } \\
\hline $\mathbf{1 a}$ & $7.83,8.47,8.98$ & $7.69,8.50,9.09$ \\
$\mathbf{1 b}$ & $8.01,8.12,8.74$ & $7.94,8.10,8.59$ \\
$\mathbf{2}$ & $8.65,9.14$ & $8.67,9.27$ \\
$\mathbf{3}$ & 8.34 & 8.38 \\
\hline
\end{tabular}

benzene ring shrinks by ca. $0.6 \AA$ on ionization of $\mathbf{1 a}, \mathbf{1 b}$, and 1c (Scheme 2), as a consequence of the formation of the $S . \therefore \pi$ bond, as described by Chen et al. ${ }^{12}$

The orbitals involved in the principal electronic transitions in $\mathbf{1 a}^{\bullet+}$ are depicted in the SI (Figure S3). The SOMO of $1 \mathbf{a}^{\mathbf{0}^{+}}$resembles the HOMO of $\mathbf{1 a}$ : it represents an antibonding combination of the sulfur p-type lone pair and a mix of the two highest $\pi$-MOs of the phenyl ring. The SOMO-1 and SOMO-2 arise from the corresponding bonding $\mathrm{S} \therefore \pi$ interaction. Therefore, the bands at 705 and $550 \mathrm{~nm}$ of the EA spectrum of $\mathbf{1 a}^{\mathbf{0}}$ (Figure 2) involve only orbitals that participate in the formation of the $[\mathrm{S} \therefore \pi]$ complex; i.e., they represent spectral signatures of that complex. As none of these MOs has any significant electronic density on the norbornyl moiety, the interaction seems to occur mainly through space.

The higher-lying transitions (bands observed at 409 and $330 \mathrm{~nm}$ ) seen in the spectrum of $\mathbf{1}^{\mathbf{}+}$ involve all charge transfer between the norbornyl and the phenyl moieties. The calculation shows a strong mixing of excitations between the different $\sigma$-orbitals participating in the transitions (see Figure $\mathrm{S} 3$ where one of the orbitals involved in the transition at $409 \mathrm{~nm}, \mathrm{SOMO}-5$, is shown).

To see whether the bands at 550 and $705 \mathrm{~nm}$ in the spectrum of $\mathbf{1}^{\bullet+}$ are really unique for the $S \therefore \pi$ interaction, the EA spectra of the radical cations of $\mathbf{2}$ and $\mathbf{3}$ were also measured (see Figure S6). The spectrum of $\mathbf{2}^{\mathbf{+}}$ shows two bands in the visible region (655 and $560 \mathrm{~nm}$ ) which, at equal concentration, are, however, much less intense than the absorption bands of $\mathbf{1}^{\mathbf{}}$. An analysis on the basis of TD-B2PLYP calculations reveals that these transitions have charge transfer character (see Figure S4). The first small band of $3^{\circ+}$ appears at $380 \mathrm{~nm}$, and it is also due to a transition from orbitals located on the norbornyl moiety to the SOMO that in $\mathbf{3}$ is localized on the sulfur atom. Thus, the absorption spectra of $\mathbf{2}^{\mathbf{}}$ and $\mathbf{3}^{\mathbf{+}}$ have nothing in common with the spectrum of $\mathbf{1}^{\bullet+}$. The detailed results of the calculations, and the main orbitals implied in the electronic transitions of both $\mathbf{2}^{\mathbf{*}}$ and $\mathbf{3}^{\mathbf{}}$, are given in the SI.

In summary, photoelectron spectra provide a good picture of the orbital interaction of a sulfur lone pair with a neighboring aromatic system. Even if a simple pairwise orbital interaction diagram does not suffice to explain the electronic structure quantitatively, it clearly shows the destabilization of the HOMO of $\mathbf{1}$ by its interaction with the phenyl ring. Furthermore, the effect of the parallel or perpendicular orientation of the methylthio group relative to the phenyl moiety can be clearly observed in the PE spectrum.

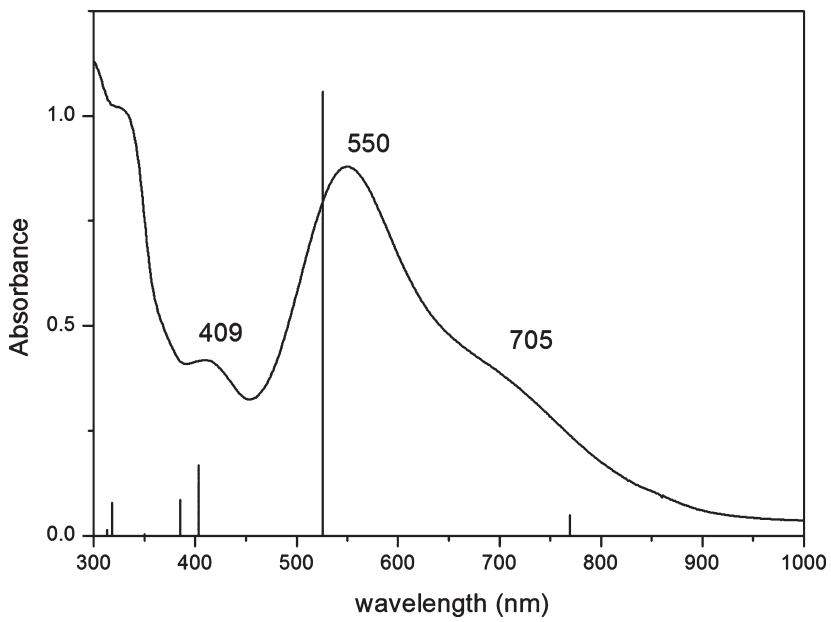

Figure 2. Difference spectrum obtained on $\gamma$-irradiation of $\mathbf{1}$ in a Freon mixture $(5 \mathrm{mM})$ at $77 \mathrm{~K}$. The bars indicate the main excitation energies and transition moments of $1 \mathbf{a}^{\cdot+}$ calculated by TD-B2PLYP

Upon one-electron oxidation, the $\mathrm{S}: \pi \pi$ interaction develops in $\mathbf{1}^{\bullet+}$. The two main observed absorption bands of $\mathbf{1}^{\mathbf{+}}$ involve orbitals resulting from the interaction of the sulfur p-type lone pair and the phenyl $\pi$-system. Therefore, these absorption bands constitute a clear spectroscopic signature of the complex. The electronic absorption spectra of $\mathbf{2}^{\mathbf{+}}$ and $3^{\cdot+}$ were also measured and confirmed that the observed absorption of $\mathbf{1}^{\boldsymbol{0}^{+}}$are characteristic of the $\mathrm{S} \therefore \pi$ complex.

In conclusion, a new bonding prototype has been experimentally substantiated, which fills the gap between $2 \mathrm{c}$, $3 \mathrm{e}$ bonding involving nonbonding electrons and the bonding between alkene, alkyne, or aromatic radical cations and arenes. ${ }^{21}$

Acknowledgment. We thank the Swiss National Foundation SNF for the financial support (Grant No. 200020143410), U.S. National Science Foundation (Grant No. 0956581), and Ms Khrystyna Regeta and Prof. Michael Allan (University of Fribourg, Switzerland) for measuring the photoelectron spectra.

Supporting Information Available. Orbital interaction diagram of $\mathbf{2}$ and $\mathbf{3}$ forming 1a (Figure S1). Molecular orbitals of $\mathbf{1 b}$ (Figure S2), $\mathbf{1 a}^{\cdot+}$ (Figure S3), $\mathbf{2}^{\mathbf{*}}$ (Figure S4), and $\mathbf{3}^{\mathbf{*}}$ (Figure S5); UV-vis spectra of $\mathbf{2}^{\mathbf{*}}$ and $\mathbf{3}^{+}$ (Figure S6); the energies of the different species (Table S1); comparison of different methods modeling the first three excited states of $1 \mathbf{a}^{\mathbf{}}$; the Cartesian coordinates and predicted electronic transitions of all optimized structures. This material is available free of charge via the Internet at http://pubs.acs.org.

(21) (a) Bally, T. In Radical Ionic Systems; Lund, A., Shiotani, M., Eds.; Kluwer: Dordrecht, 1991; pp 1-55. (b) Kochi, J. K.; Rathore, R.; Le Magueres, P. J. Org. Chem. 2000, 65, 6826-6836. (c) Das, T. N. J. Phys. Chem. A 2009, 113, 6489-6493.

The authors declare no competing financial interest. 